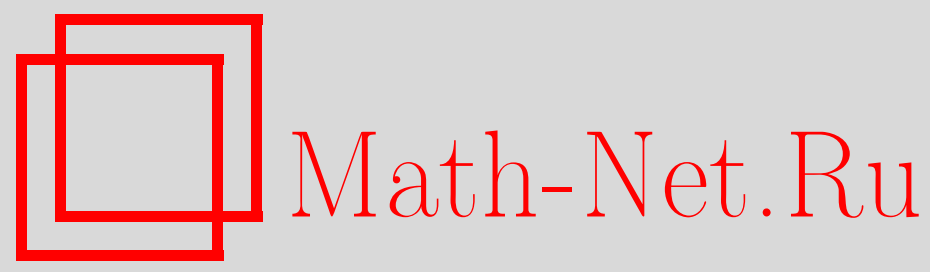

В. В. Галатенко, Е. Д. Лившиц, Обобщенные приближенные слабые жадные алгоритмы, Матем. заметки, 2005, том 78, выпуск 2, 186-201

DOI: https://doi.org/10.4213/mzm2581

Использование Общероссийского математического портала Math-Net.Ru подразумевает, что вы прочитали и согласны с пользовательским соглашением http://www . mathnet.ru/rus/agreement

Параметры загрузки:

IP: 54.237 .206 .68

26 апреля 2023 г., 15:58:12

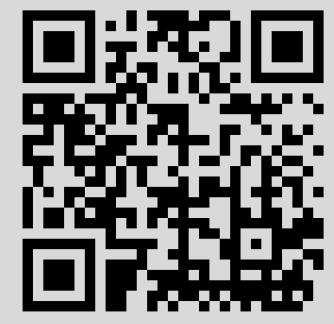




\title{
ОБОБЩЕННЫЕ ПРИБЛИЖЕННЫЕ СЛАБЫЕ ЖАДНЫЕ АЛГОРИТМЫ
}

\author{
В. В. Галатенко, Е. Д. Лившиц
}

Рассмотрены обобщенные приближенные слабые жадные алгоритмы. Основное отличие этих алгоритмов от предложенных Р. Грибонвалем и М. Нилсеном приближенных слабых жадных алгоритмов заключается в том, что ошибки в вычислении коэффициентов можно задавать не только через их относительные, но и через их абсолютные величины. Приведены условия на параметры обобщенных приближенных слабых жадных алгоритмов, достаточные для сходимости полученных в результате применения этого алгоритма разложений к разлагаемому элементу. Показано, что эти условия нельзя существенно ослабить. Исследованы некоторые вопросы сходимости обобщенных приближенных слабых жадных разложений по ортонормированным системам.

Библиография: 10 названий.

1. Введение. Приближенные слабые жадные алгоритмы (AWGA - Approximate Weak Greedy Algorithms), предложенные Грибонвалем и Нилсеном в работе [1], описьвают процесс жадных разложений с ошибками в вычислении коэффициентов, характеризуя ошибку ее относительной величиной. Представляется естественньм наравне с относительньми ошибками рассматривать также абсолютные ошибки.

Начнем с того, что дадим необходимые определения. Пусть $(H,(\cdot, \cdot))$ - гильбертово пространство над полем действительных чисел, множество $D$ элементов $H-c л о в а р ь ~$ (dictionary), т.е. для любого элемента $d \in D$ справедливо равенство $\|d\|=1$ и замыкание линейной оболочки $D$ совпадает с $H$. Пусть также $f$ - некоторый элемент $H$, $\left\{t_{n}\right\}_{n=1}^{\infty} \subset[0,1]-$ последовательность чисел из отрезка $[0,1],\left\{q_{n}\right\}_{n=1}^{\infty} \subset[0, \infty)$-последовательность действительных неотрищательных чисел, $\left\{\left(\varepsilon_{n}, \xi_{n}\right)\right\}_{n=1}^{\infty}-$ последовательность числовых пар. Определим индуктивно последовательность остатков $\left\{r_{n}(f)\right\}_{n=0}^{\infty}$, последовательность коэффищиентов $\left\{\widehat{f}_{n}\right\}_{n=1}^{\infty}$ и последовательность разлагающих элементов $\left\{e_{n}(f)\right\}_{n=1}^{\infty}$. Положим

$$
r_{0}(f)=f
$$

Далее, если уже определен остаток $r_{n}(f), n \geqslant 0$, найдем элемент $e_{n+1}(f) \in D$, удовлетворяюший условию

$$
\left|\left(r_{n}(f), e_{n+1}(f)\right)\right| \geqslant t_{n+1} \sup _{e \in D}\left|\left(r_{n}(f), e\right)\right|-q_{n+1}
$$

Работа выполнена при финансовой поддержке Российского фонда фундаментальных исследований, грант № 05-01-00062, и программы “Ведушие научные шшколы Р Ф”, грант № НШ-1657.2003.1. 
(Если элементов, удовлетворяюших этому условию, несколько, в качестве $e_{n+1}(f)$ выберем любой из них. Если элементов, удовлетворяюших этому условию, не существует, будем говорить, что осуществить разложение не удалось. Отметим, что последний случай возможен, только если $t_{n+1}=1$ и $q_{n+1}=0$.) Положим

$$
\begin{gathered}
\widehat{f}_{n+1}=\left(r_{n}(f), e_{n+1}(f)\right)\left(1+\varepsilon_{n+1}\right)+\xi_{n+1}, \\
r_{n+1}(f)=r_{n}(f)-\widehat{f}_{n+1} e_{n+1}(f)=f-\sum_{k=1}^{n+1} \widehat{f}_{k} e_{k}(f) .
\end{gathered}
$$

ОПРедЕЛЕниЕ 1. Описанньй вьше процесс будем называть обобщенным приближенным слабым жадным алгоритмом (gAWGA - generilized Approximate Weak Greedy Algorithm). Если разложение удалось осуществить, формальный ряд $\sum_{n=1}^{\infty} \widehat{f}_{n} e_{n}(f)$ будем назьвать gAWGA-разложением элемента $f$ по словарю $D$ с ослабляющими последовательностями $\left\{t_{n}\right\}_{n=1}^{\infty},\left\{q_{n}\right\}_{n=1}^{\infty}$ и последовательностью ошибок $\left\{\left(\varepsilon_{n}, \xi_{n}\right)\right\}_{n=1}^{\infty}$.

Последовательность ошибок $\left\{\left(\varepsilon_{n}, \xi_{n}\right)\right\}_{n=1}^{\infty}$ допускает следующую естественную интерпретацию: если $\left|\varepsilon_{n}\right|$ мал, то $\xi_{n}$ можно рассматривать как число, характеризующее абсолютную величину ошибки в вычислении коэффициента $\widehat{f}_{n}$; если $\left|\xi_{n}\right|$ мал, то $\varepsilon_{n}$ можно рассматривать как число, характеризующее относительную величину ошибки в вычислении коэффициента $\widehat{f}_{n}, n=1,2,3, \ldots$.

Различньм парам $\left(\varepsilon_{n}, \xi_{n}\right)$ может соответствовать одна и та же ошибка в вычислении коэффициента $\widehat{f}_{n}$. В то же время, любая ошибка в вычислении $\widehat{f}_{n}$ может быть выражена посредством пары вида $\left(0, \xi_{n}\right)$. Однако используемые для записи вычислительных ошибок обозначения представляются удобными для практических применений, так как при реализации вычислений в некоторых случаях проще получать оценки на относительные величины ошибок, а в некоторых - на абсолютные.

Если $q_{n}=\xi_{n}=0$ для всех натуральных $n$, то gAWGA совпадает с AWGA. Если для всех натуральных $n$ выполняются равенства $q_{n}=\xi_{n}=0, \varepsilon_{n}=0$, то gAWGA совпадает со слабым жадным алгоритмом (WGA - Weak Greedy Algorithm; cм. [2]). Если $q_{n}=\xi_{n}=\varepsilon_{n}=0$ и $t_{n}=1$ для всех натуральных $n$, то gAWGA совпадает с чистым жадным алгоритмом (PGA - Pure Greedy Algorithm), известным так же как Projection Pursuit Regression и Matching Pursuit (см. [3], [4]).

Сходимость к разлагаемому элементу PGA-разложений, а также WGA-разложений с $t_{n} \geqslant t, 0<t<1$, была установлена в работах Джонса [5], [6] и в работе Рейте и Уолтера [7]. Первое условие на последовательность $\left\{t_{n}\right\}_{n=1}^{\infty} \mathrm{c} \liminf _{n \rightarrow \infty} t_{n}=0$, достаточное для сходимости WGA-разложений, было получено Темляковым в статье [2]. Им было показано, что если ряд $\sum_{n=1}^{\infty}\left(t_{n} / n\right)$ расходится, то для любого гильбертова пространства $H$ и любого словаря $D \subset H$ WGA-разложение любого элемента $f \in H \mathrm{c}$ ослабляющей последовательностью $\left\{t_{n}\right\}_{n=1}^{\infty}$ сходится к $f$. Первое нетривиальное условие на $\left\{t_{n}\right\}_{n=1}^{\infty}$, необходимое для сходимости всех WGA-разложений с такой ослабляющей последовательностью, было получено Лившищем и Темляковым в работе [8]. Позднее Темляков получил условие на $\left\{t_{n}\right\}_{n=1}^{\infty}$, необходимое и достаточное для того, чтобы для любого гильбертова $H$, любого словаря $D \subset H$ и любого элемента $f \in H$ WGA-разложение элемента $f$ с ослабляющей последовательностью $\left\{t_{n}\right\}_{n=1}^{\infty}$ сходилось к $f$ (см. [9]). Сходимость AWGA-разложений изучалась Грибонвалом и Нилсеном в статье [1]. В этой работе ими, в частности, было показано, что если для некоторого $\delta>0$ 
$\left\{\varepsilon_{n}\right\}_{n=1}^{\infty} \subset[-1,1-\delta]$ и ряд

$$
\sum_{n=1}^{\infty} \frac{t_{n}\left(1-\varepsilon_{n}^{2}\right)}{n}
$$

расходится, то AWGA-разложение сходится к разлагаемому элементу. Этот результат был немного улучшен в статье [10], в которой была доказана следуюшая теорема.

Tеорема 1 [10]. Пусть для некоторого $\delta>0 \quad\left\{\varepsilon_{n}\right\}_{n=1}^{\infty} \subset[-1,1-\delta] u$

$$
\sum_{n=1}^{\infty} \frac{t_{n}\left(1-\varepsilon_{n}^{2}\right)^{1 / 2}}{n}=\infty
$$

Тогда для любого гильбертова пространства $H$, любого словаря $D \subset H$ и любого әлемента $f \in H$ AWGA-разлохсение $f$ по системе $D$ с ослабляющей последовательностью $\left\{t_{n}\right\}_{n=1}^{\infty}$ и относительными ошибками $\left\{\varepsilon_{n}\right\}_{n=1}^{\infty}$ сходится $\kappa f$.

В настояшей работе доказьвается аналогичньй результат для gAWGA-разложений. Кроме того, изучаются вопросы возможности ослабления полученных условий, а также исследуется сходимость gAWGA-разложений по ортонормированным системам с параметрами $q_{n}=\varepsilon_{n}=0, t_{n} \geqslant t$, где $t \in(0,1], n=1,2,3, \ldots$

2. Достаточные условия сходимости gAWGA-разложений. В этом пункте формулируется и доказывается обобшение теоремы 1 , а также показывается, что входящие в данное обобщение условия на последовательности $\left\{\xi_{n}\right\}_{n=1}^{\infty}$ и $\left\{q_{n}\right\}_{n=1}^{\infty}$ не могут быть значительно ослаблены. При этом рассматриваются гильбертовы пространства не только над полем действительных, но и над полем комплексных чисел (определение gAWGA-разложений в гильбертовых пространствах над $\mathbb{C}$ в точности такое же, как и в гильбертовых пространствах над $\mathbb{R})$.

ТЕОРема 2. Пусть для некоторых чисел $\delta \in(0,1)$ и $C>0$ выполняются следующие условия:

$$
\left|\varepsilon_{n}+\delta\right|<1-\delta, \quad\left|\operatorname{Im} \varepsilon_{n}\right| \leqslant C\left(\operatorname{Re} \varepsilon_{n}+1\right), \quad n=1,2,3, \ldots
$$

Пусть также

$$
\begin{gathered}
\sum_{n=1}^{\infty} \frac{t_{n}\left(1-\left|\varepsilon_{n}\right|^{2}\right)^{1 / 2}}{n}=\infty \\
\sum_{n=1}^{\infty} \frac{\left|\xi_{n}\right|^{2}}{1-\left|\varepsilon_{n}\right|^{2}}<\infty, \\
\sum_{n=1}^{\infty} q_{n}^{2}\left(1-\left|\varepsilon_{n}\right|^{2}\right)<\infty .
\end{gathered}
$$

Тогда для любого гильбертова пространства $H$, любого словаря $D \subset H$ и любого әлемента $f \in H$ gAWGA-разложение $f$ по $D$ с ослабляющими последовательностям $и\left\{t_{n}\right\}_{n=1}^{\infty},\left\{q_{n}\right\}_{n=1}^{\infty}$ и ошибками $\left\{\left(\varepsilon_{n}, \xi_{n}\right)\right\}_{n=1}^{\infty}$ сходится $\kappa f$. 
ДокАЗАТЕЛьСтво. Зафиксируем произвольное гильбертово пространство $H$, произвольный словарь $D \subset H$ и произвольньй элемент $f \in H$. Для упрощения формул разлагаюшие элементы и остатки gAWGA-разложения элемента $f$ по словарю $D$ с ослабляюшими последовательностями $\left\{t_{n}\right\}_{n=1}^{\infty},\left\{q_{n}\right\}_{n=1}^{\infty}$ и ошибками $\left\{\left(\varepsilon_{n}, \xi_{n}\right)\right\}_{n=1}^{\infty}$ будем обозначать через $e_{n}$ и $r_{n}$ соответственно.

Можно считать, что

$$
q_{n} \leqslant t_{n} \sup _{e \in D}\left|\left(r_{n-1}(f), e\right)\right|
$$

для всех $n \in \mathbb{N}$. Действительно, если это неравенство для некоторых $n$ не выполняется, то заменим $\left\{q_{n}\right\}_{n=1}^{\infty}$ на

$$
\left\{\min \left\{q_{n}, t_{n} \sup _{e \in D}\left|\left(r_{n-1}(f), e\right)\right|\right\}\right\}_{n=1}^{\infty}
$$

Это изменение не влияет на выбор разлагающих элементов и, таким образом, не меняет разложение.

При доказательстве теоремы 2 используется техника, разработанная Темляковым (см. [2]). Эта техника была также использована в работах [1], [10]. Начнем доказательство с некоторых лемм.

ЛЕмма 1. Для всех иелых неотрицательных чисел $n$ справедливо следующее равенство:

$$
\left\|r_{n+1}\right\|^{2}=\left\|r_{n}\right\|^{2}-\left(1-\left|\varepsilon_{n+1}\right|^{2}\right)\left|\left(r_{n}, e_{n+1}\right)-\frac{\overline{\varepsilon_{n+1}} \xi_{n+1}}{1-\left|\varepsilon_{n+1}\right|^{2}}\right|^{2}+\frac{\left|\xi_{n+1}\right|^{2}}{1-\left|\varepsilon_{n+1}\right|^{2}}
$$

(черта означает комплексное сопряжение).

ДокАЗАТЕЛЬСТво. Из формул (3) и (4) следует, что для всех $n \in\{0,1,2, \ldots\}$ выполняется равенство

$$
r_{n+1}=r_{n}-\left(\left(r_{n}, e_{n+1}\right)\left(1+\varepsilon_{n+1}\right)+\xi_{n+1}\right) e_{n+1} .
$$

Вычислив скалярные квадраты левой и правой частей этого равенства, получаем соотношение

$$
\begin{aligned}
\left\|r_{n+1}\right\|^{2}= & \left\|r_{n}\right\|^{2}-\left(1-\left|\varepsilon_{n+1}\right|^{2}\right)\left|\left(r_{n}, e_{n+1}\right)\right|^{2} \\
& +\left(r_{n}, e_{n+1}\right) \varepsilon_{n+1} \overline{\xi_{n+1}}+\overline{\left(r_{n}, e_{n+1}\right) \varepsilon_{n+1}} \xi_{n+1}+\left|\xi_{n+1}\right|^{2} .
\end{aligned}
$$

Для завершения доказательства леммы остается преобразовать последнее соотношение, учитывая, что справедливо неравенство $\left|\varepsilon_{n+1}\right|<1$ и, значит, выражение $1-\left|\varepsilon_{n+1}\right|^{2}$ не равно нулю.

СлЕДСТВИЕ 1. Для всех целых неотрицательных $n$ справедливо тождество

$$
\|f\|^{2}-\left\|r_{n}\right\|^{2}=\sum_{k=1}^{n}\left(\left(1-\left|\varepsilon_{k}\right|^{2}\right)\left|\left(r_{k-1}, e_{k}\right)-\frac{\overline{\varepsilon_{k}} \xi_{k}}{1-\left|\varepsilon_{k}\right|^{2}}\right|^{2}-\frac{\left|\xi_{k}\right|^{2}}{1-\left|\varepsilon_{k}\right|^{2}}\right)
$$

(сумму из нуля слагаемых считаем равной нулю), и, следовательно,

$$
\sum_{k=1}^{\infty}\left(1-\left|\varepsilon_{k}\right|^{2}\right)\left|\left(r_{k-1}, e_{k}\right)-\frac{\overline{\varepsilon_{k}} \xi_{k}}{1-\left|\varepsilon_{k}\right|^{2}}\right|^{2} \leqslant \sum_{k=1}^{\infty} \frac{\left|\xi_{k}\right|^{2}}{1-\left|\varepsilon_{k}\right|^{2}}+\|f\|^{2}<\infty .
$$


ЛЕмма 2. Последовательность квадратов норм $\left\{\left\|r_{n}\right\|^{2}\right\}_{n=1}^{\infty}$ сходится.

ДокАЗАТЕЛЬСТвО. Из леммы 1 следует, что для всех целых неотрицательных $m$ и $n$, $m<n$, справедливо равенство

$$
\left\|r_{n}\right\|^{2}-\left\|r_{m}\right\|^{2}=\sum_{k=m+1}^{n}\left(\left(1-\left|\varepsilon_{k}\right|^{2}\right)\left|\left(r_{k-1}, e_{k}\right)-\frac{\overline{\varepsilon_{k}} \xi_{k}}{1-\left|\varepsilon_{k}\right|^{2}}\right|^{2}-\frac{\left|\xi_{k}\right|^{2}}{1-\left|\varepsilon_{k}\right|^{2}}\right) .
$$

Используя это равенство, а также неравенства (7) и (9), получаем, что $\left\{\left\|r_{n}\right\|^{2}\right\}_{n=1}^{\infty}$ является последовательностью Коши и, значит, является сходящейся последовательностью.

Лемма 3. Существует такая строго возрастающая последовательность натуральных чисел $\left\{n_{k}\right\}_{k=1}^{\infty}$, что последовательность $\left\{r_{n_{k}}\right\}_{k=1}^{\infty}$ слабо сходится $\kappa$ нулю.

ДокАЗАТЕЛЬСТво. Используя неравенство (9), а также учитывая, что согласно условиям (5) и (7)

$$
\left\{\frac{\overline{\varepsilon_{k}} \xi_{k}}{\sqrt{1-\left|\varepsilon_{k}\right|^{2}}}\right\}_{k=1}^{\infty} \in \ell^{2}
$$

получаем соотношение

$$
\sum_{k=1}^{\infty}\left(1-\left|\varepsilon_{k}\right|^{2}\right)\left|\left(r_{k-1}, e_{k}\right)\right|^{2}<\infty
$$

Из соотношений (2) и (10) следует, что

$$
\sum_{k=1}^{\infty}\left(1-\left|\varepsilon_{k}\right|^{2}\right)\left(t_{k} \sup _{e \in D}\left|\left(r_{k-1}, e\right)\right|-q_{k}\right)^{2} \leqslant \sum_{k=1}^{\infty}\left(1-\left|\varepsilon_{k}\right|^{2}\right)\left|\left(r_{k-1}, e_{k}\right)\right|^{2}<\infty .
$$

Используя последнее неравенство и условие (8), получаем

$$
\sum_{k=1}^{\infty}\left(1-\left|\varepsilon_{k}\right|^{2}\right) t_{k}^{2} \sup _{e \in D}\left|\left(r_{k-1}, e\right)\right|^{2}<\infty
$$

Но из условия (6) и неравенства Коши-Буняковского-Шварца следует, что

$$
\sum_{k=1}^{\infty} t_{k}^{2}\left(1-\left|\varepsilon_{k}\right|^{2}\right)=\infty
$$

Следовательно, сушествует подпоследовательность $\left\{r_{n_{k}}\right\}_{k=1}^{\infty}$, удовлетворяющая условию

$$
\sup _{e \in D}\left|\left(r_{n_{k}}, e\right)\right| \rightarrow 0, \quad k \rightarrow \infty .
$$

Из этого утверждения и равенства $\overline{\operatorname{span}} D=H$ непосредстванно вытекает утверждение леммы.

Следующая лемма была доказана Конягиным и Темляковым (см. [2, лемма 2.3]). 
Лемма 4 (С.В. Конягин, В.Н. Темляков). Пусть последовательность $\left\{\tau_{k}\right\}_{k=1}^{\infty}$ удовлетворяет условиям

$$
\tau_{k} \geqslant 0, \quad k=1,2,3, \ldots, \quad \sum_{k=1}^{\infty} \frac{\tau_{k}}{k}=\infty
$$

Пусть также последовательность $\left\{\alpha_{k}\right\}_{k=1}^{\infty} \in \ell^{2}$. Тогда имеет место равенство

$$
\liminf _{n \rightarrow \infty} \frac{\left|\alpha_{n}\right|}{\tau_{n}} \sum_{k=1}^{n}\left|\alpha_{k}\right|=0
$$

(если $\tau_{n}=0$ для некоторых $n$, считаем, что для әтих $n$ выражение $\left(\left|\alpha_{n}\right| / \tau_{n}\right) \times$ $\sum_{k=1}^{n}\left|\alpha_{k}\right|$ равно бесконечности).

Отметим, что в статье [2] эта лемма была доказана только для случая $\tau_{k}>0, k=$ $1,2,3, \ldots$, но в случае $\tau_{k} \geqslant 0$ проходит то же доказательство с незначительньми изменениями.

Лемма 5. Существуют такие числа $C_{1}>0$ и $C_{2}>0$, что неравенства

$$
C_{1}\left|1+\varepsilon_{n}\right| \leqslant 1-\left|\varepsilon_{n}\right|^{2} \leqslant C_{2}\left|1+\varepsilon_{n}\right|
$$

справедливы для всех $n \in \mathbb{N}$.

ДокАЗАТЕльСтво. Вьведем искомое утверждение из условия (5). В случае поля действительных чисел это тривиально: условие (5) дает, что для некоторого $\delta \in(0,1)$ справедливо неравенство $-1<\varepsilon_{n}<1-2 \delta$, откуда немедленно следует оценка

$$
2 \delta\left(1+\varepsilon_{n}\right)<1-\varepsilon_{n}^{2}<2\left(1+\varepsilon_{n}\right),
$$

т.е. в качестве $C_{1}$ можно брать число $2 \delta$, а в качестве $C_{2}-$ двойку.

Комплексньй случай несколько сложнее. Пусть

$$
K=\{z \in \mathbb{C}:|z+\delta|<1-\delta,|\operatorname{Im} z| \leqslant C(\operatorname{Re} z+1)\}
$$

(числа $\delta$ и $C$ здесь те же, что и в условии (5)). Рассмотрим на множестве $K$ функцию

$$
\varphi(z)=\frac{1-|z|^{2}}{|1+z|}
$$

Достаточно доказать, что существуют положительные числа $C_{1}$ и $C_{2}$ такие, что для всех $z \in K$ справедлива оценка $C_{1} \leqslant \varphi(z) \leqslant C_{2}$.

Зафиксируем произвольное число $z \in K$ и запишем его в виде $z=-1+x+i y$. Тогда значение функции $\varphi$ в точке $z$ запишется следующим образом:

$$
\varphi(z)=\frac{2 x-x^{2}-y^{2}}{\sqrt{x^{2}+y^{2}}} .
$$


Справедливы следующие оценки:

$$
\frac{2 x-x^{2}-C^{2} x^{2}}{\sqrt{x^{2}+C^{2} x^{2}}} \leqslant \varphi(z) \leqslant \frac{2 x}{\sqrt{x^{2}}} .
$$

Из этих оценок следует, что существует такое число $\gamma>0$, что для любого $z \in K \cap$ $\{|1+z|<\gamma\}$ выполняется неравенство

$$
\frac{1}{\sqrt{1+C^{2}}} \leqslant \varphi(z) \leqslant 2 .
$$

Остается заметить, что на замькании множества $K_{1}=K \cap\{|1+z| \geqslant \gamma\}$ функция $\varphi(z)$ является непрерьвной функцией, принимающей лишь положительные значения. Отсюда в силу компактности замыкания $K_{1}$ следует, что существуют такие положительные числа $c_{1}$ и $c_{2}$, что для всех $z \in K_{1}$ справедливо соотношение $c_{1} \leqslant \varphi(z) \leqslant c_{2}$. Таким образом, утверждение леммы вьполняется с константами

$$
C_{1}=\min \left\{\frac{1}{\sqrt{1+C^{2}}}, c_{1}\right\}, \quad C_{2}=\max \left\{2, c_{2}\right\} .
$$

Перейдем непосредственно к доказательству теоремы. Необходимо показать, что $r_{n} \rightarrow 0$ при $n \rightarrow \infty$. Для этого, учитьвая леммы 2,3 и тождество

$$
\left\|r_{n}-r_{m}\right\|^{2}=\left\|r_{n}\right\|^{2}-\left\|r_{m}\right\|^{2}-\left(r_{n}-r_{m}, r_{m}\right)-\left(r_{m}, r_{n}-r_{m}\right),
$$

достаточно доказать следующее утверждение:

$$
\forall \tilde{\delta}>0, \quad \forall M \in \mathbb{N} \quad \exists m>M: \quad \forall n<m \quad\left|\left(r_{n}-r_{m}, r_{m}\right)\right|<\tilde{\delta} .
$$

Используя равенство $r_{m}-r_{n}=\sum_{k=n+1}^{m} \widehat{f}_{k} e_{k}$, формулы (2)-(4), а также неравенство $x \leqslant(2 x)^{1 / 2}, 0 \leqslant x \leqslant 2$, получаем оценку

$$
\begin{aligned}
\left|\left(r_{n}-r_{m}, r_{m}\right)\right| \leqslant & \sum_{k=n+1}^{m}\left|\widehat{f}_{k}\right|\left|\left(r_{m}, e_{k}\right)\right| \leqslant \sup _{e \in D}\left|\left(r_{m}, e\right)\right| \sum_{k=n+1}^{m}\left|\widehat{f}_{k}\right| \\
\leqslant & \frac{\left|\left(r_{m}, e_{m+1}\right)\right|+q_{m+1}}{t_{m+1}} \sum_{k=1}^{m+1}\left|\left(1+\varepsilon_{k}\right)\left(r_{k-1}, e_{k}\right)+\xi_{k}\right| \\
\leqslant & 2^{1 / 2} \frac{\left|\left(r_{m}, e_{m+1}\right)\right|\left|1+\varepsilon_{m+1}\right|^{1 / 2}+q_{m+1}\left|1+\varepsilon_{m+1}\right|^{1 / 2}+\left|\xi_{m+1}\right|}{t_{m+1}\left|1+\varepsilon_{m+1}\right|^{1 / 2}} \\
& \times \sum_{k=1}^{m+1}\left(\left|1+\varepsilon_{k}\right|^{1 / 2}\left|\left(r_{k-1}, e_{k}\right)\right|+\left|\xi_{k}\right|+q_{k}\left|1+\varepsilon_{k}\right|^{1 / 2}\right) .
\end{aligned}
$$

Для завершения доказательства теоремы остается применить лемму 4 , положив

$$
\tau_{k}=t_{k}\left|1+\varepsilon_{k}\right|^{1 / 2}, \quad \alpha_{k}=\left|1+\varepsilon_{k}\right|^{1 / 2}\left|\left(r_{k-1}, e_{k}\right)\right|+\left|\xi_{k}\right|+q_{k}\left|1+\varepsilon_{k}\right|^{1 / 2}
$$

(тот факт, что такие последовательности $\left\{\tau_{k}\right\}_{k=1}^{\infty}$ и $\left\{\alpha_{k}\right\}_{k=1}^{\infty}$ удовлетворяют условиям леммы 4 , следует из условий (6)-(8), соотношения (10) и леммы 5$)$.

Условия (5) и (6) теоремы 2 появились в работах [1] и [10], где и обсуждались некоторые вопросы, связанные с возможностью ослабления этих условий. Что касается новых условий, т.е. условий (7) и (8), то ниже будет показано, что существенно их ослабить нельзя. Точнее, справедливы следующие теоремы. 
Tеорема 3. Существуют гильбертово пространство $H$, словарь $D \subset H$, әлемент $f \in H$ и последовательность $\left\{\xi_{n}\right\}_{n=1}^{\infty}$ такие, что для некоторого положительного числа С справедливы неравенства

$$
\left|\xi_{n}\right| \leqslant \frac{C}{\sqrt{n}}, \quad n=1,2,3, \ldots,
$$

$u$ gAWGA-разложение әлемента $f$ по словарю $D$ с ослабляющими последовательностями $t_{n}=1, q_{n}=0, n=1,2,3, \ldots$, и ошибками $\left\{\left(0, \xi_{n}\right)\right\}_{n=1}^{\infty}$ не сходится $\kappa f$.

Tеорема 4. Существуют гильбертово пространство $H$, словарь $D \subset H$, әлемент $f \in H$ и последовательность $\left\{q_{n}\right\}_{n=1}^{\infty}$ такие, что для некоторого положительного числа $C$ справедливы неравенства

$$
q_{n} \leqslant \frac{C}{\sqrt{n}}, \quad n=1,2,3, \ldots
$$

$u$ gAWGA-разложение әлемента $f$ по словарю $D$ с ослабляющими последовательностями $t_{n}=1, n=1,2,3, \ldots,\left\{q_{n}\right\}_{n=1}^{\infty}$ и ошибками $\varepsilon_{n}=\xi_{n}=0, n=1,2,3, \ldots$, не сходится $\kappa f$.

ДокАЗАТЕЛЬСтво теОРЕмЫ 3. Рассмотрим бесконечномерное действительное сепарабельное гильбертово пространство $H$. Пусть $\left\{h_{n}\right\}_{n=1}^{\infty}$ - ортонормированный базис в $H, N$ - некоторое натуральное число, $N \geqslant 2$. Для $n \geqslant N$ рассмотрим векторы

$$
f_{n}=\left(\frac{1}{n^{1 / 2}}+\frac{1}{n}\right) \sum_{k=1}^{n} h_{k}
$$

и положим

$$
c_{n}=\left\|f_{n-1}-f_{n}\right\|, \quad g_{n}=\frac{1}{c_{n}}\left(f_{n-1}-f_{n}\right) .
$$

Из определения $c_{n}$ и $g_{n}$ непосредственно следует, что $\left\|g_{n}\right\|=1$ и справедливо равенство

$$
f_{n}=f_{n-1}-c_{n} g_{n}
$$

Введем следуюшее обозначение. Для последовательности $\left\{y_{n}\right\}_{n=N}^{\infty}$ будем писать

$$
y_{n} \simeq b n^{\alpha}+c n^{\alpha-1 / 2}, \quad b \neq 0,
$$

если для некоторого действительного числа $d$

$$
y_{n}=b n^{\alpha}\left(1+\frac{c / b}{n^{1 / 2}}+\frac{d}{n}+O\left(\frac{1}{n^{3 / 2}}\right)\right), \quad n \rightarrow \infty .
$$

В частности, мы будем писать

$$
y_{n} \simeq b n^{\alpha}, \quad b \neq 0,
$$


если для некоторого действительного числа $d$

$$
y_{n}=b n^{\alpha}\left(1+\frac{d}{n}+O\left(\frac{1}{n^{3 / 2}}\right)\right), \quad n \rightarrow \infty .
$$

Используя равенство (12), для $n \geqslant N+1$ получаем, что

$$
f_{n-1}-f_{n}=\left(\frac{1}{(n-1)^{1 / 2}}-\frac{1}{n^{1 / 2}}+\frac{1}{n-1}-\frac{1}{n}\right) \sum_{k=1}^{n-1} h_{k}-\left(\frac{1}{n^{1 / 2}}+\frac{1}{n}\right) h_{n} .
$$

Отсюда следует, что

$$
c_{n}^{2}=(n-1)\left(\frac{1}{(n-1)^{1 / 2}}-\frac{1}{n^{1 / 2}}+\frac{1}{n-1}-\frac{1}{n}\right)^{2}+\frac{(1+1 / \sqrt{n})^{2}}{n} \simeq \frac{1}{n}+\frac{2}{n^{3 / 2}}
$$

и, значит,

$$
c_{n} \simeq \frac{1}{n^{1 / 2}}+\frac{1}{n} .
$$

Векторы $g_{n}, n \geqslant N+1$, могут быть представлены следующим образом:

$$
g_{n}=\alpha_{n} \sum_{k=1}^{n-1} h_{k}+\beta_{n} h_{n}
$$

где

$$
\begin{aligned}
\alpha_{n} & =\frac{1}{c_{n}}\left(f_{n-1}-f_{n}, h_{n-1}\right) \\
& =\left(\frac{1}{(n-1)^{1 / 2}}-\frac{1}{n^{1 / 2}}+\frac{1}{n-1}-\frac{1}{n}\right)\left(\frac{1}{n^{1 / 2}}+\frac{1}{n}+\frac{d_{1}}{n^{3 / 2}}+O\left(\frac{1}{n^{2}}\right)\right)^{-1} \\
& =\left(\frac{1 / 2}{n^{3 / 2}}+\frac{1}{n^{2}}+\frac{d_{2}}{n^{5 / 2}}+O\left(\frac{1}{n^{3}}\right)\right)\left(\frac{1}{n^{1 / 2}}+\frac{1}{n}+\frac{d_{1}}{n^{3 / 2}}+O\left(\frac{1}{n^{2}}\right)\right)^{-1} \\
& =\left(\frac{1 / 2}{n}\right) \frac{1+2 / n^{1 / 2}+2 d_{2} / n+O\left(1 / n^{3 / 2}\right)}{1+1 / n^{1 / 2}+d_{1} / n+O\left(1 / n^{3 / 2}\right)} \simeq \frac{1 / 2}{n}+\frac{1 / 2}{n^{3 / 2}} .
\end{aligned}
$$

Тогда

$$
\begin{aligned}
\left(f_{n-1}, g_{n}\right) & =(n-1)\left(\frac{1}{(n-1)^{1 / 2}}+\frac{1}{n-1}\right) \alpha_{n} \\
& =\left(n^{1 / 2}+1+\left((n-1)^{1 / 2}-n^{1 / 2}\right)\right) \alpha_{n} \simeq \frac{1 / 2}{n^{1 / 2}}+\frac{1}{n} .
\end{aligned}
$$

Для $n \geqslant N+1$ определим числа

$$
\xi_{n-N}:=-\left(f_{n}, g_{n}\right)=-\left(f_{n-1}-c_{n} g_{n}, g_{n}\right)=c_{n}-\left(f_{n-1}, g_{n}\right)
$$

Учитьвая соотношения (13) и (14), получаем, что

$$
\xi_{n-N}=c_{n}-\left(f_{n-1}, g_{n}\right) \simeq \frac{1 / 2}{n^{1 / 2}} .
$$


Выберем число $N$ настолько большим, что для всех натуральных чисел $m, n$ таких, что $N<n<m$, вьполняются неравенства

$$
\begin{aligned}
& \alpha_{n}>\alpha_{m}>0 \\
& \xi_{n-N}<\frac{1 / 2}{n^{1 / 2}}+\frac{1 / 2}{n}, \\
& \frac{1 / 2}{(n-1)^{1 / 2}}+\frac{1 / 2}{n-1}<\left(f_{n-1}, g_{n}\right) .
\end{aligned}
$$

Далее выберем такую произвольную нормированную систему $\left\{g_{l}\right\}_{l=1}^{N} \in H$, что

$$
\begin{gathered}
\operatorname{span}_{l=1}^{N}\left\{g_{l}\right\}=\operatorname{span}_{l=1}^{N}\left\{h_{l}\right\}, \\
\left|\left(g_{l}, f_{N}\right)\right|<\frac{1 / 4}{N^{1 / 2}}, \quad l=1, \ldots, N
\end{gathered}
$$

Легко видеть, что система $D=\left\{g_{k}\right\}_{k=1}^{\infty}$ является словарем.

Докажем, что gAWGA-разложение элемента $f:=f_{N}$ по словарю $D$ с ослабляющими последовательностями $t_{n}=1, q_{n}=0, n=1,2,3, \ldots$, и ошибками $\left\{\left(0, \xi_{n}\right)\right\}_{n=1}^{\infty}$ расходится. Пусть $\left\{r_{n}(f)\right\}_{n=0}^{\infty}$ и $\left\{e_{n}(f)\right\}_{n=1}^{\infty}-$ соответственно последовательность остатков и последовательность разлагающих элементов рассматриваемого gAWGA-разложения. Покажем, что для каждого $j \geqslant 0$ вьполняются следуюшие соотношения:

$$
r_{j}(f)=f_{N+j}, \quad \max _{1 \leqslant l \leqslant N+j}\left|\left(f_{N+j}, g_{l}\right)\right| \leqslant \frac{1 / 2}{(N+j)^{1 / 2}}+\frac{1 / 2}{N+j} .
$$

Доказательство проведем индукцией по $j$. При $j=0$ искомое утверждение непосредственно вытекает из неравенств (20). Предположим, что искомое утверждение справедливо для $j=k$, и покажем, что оно верно для $j=k+1$.

Представим число $k$ в виде $k=n-N-1, n \geqslant N+1$. Используя неравенства (17), (19), получим, что для всех натуральных $m, m>n$, справедлива оценка

$$
\begin{aligned}
\left|\left(r_{k}(f), g_{m}\right)\right| & =\left|\left(f_{n-1}, g_{m}\right)\right|=\left(\frac{1}{(n-1)^{1 / 2}}+\frac{1}{n-1}\right)(n-1) \alpha_{m} \\
& <\left(\frac{1}{(n-1)^{1 / 2}}+\frac{1}{n-1}\right)(n-1) \alpha_{n}=\left|\left(f_{n-1}, g_{n}\right)\right|=\left|\left(r_{k}(f), g_{n}\right)\right|
\end{aligned}
$$

и согласно соотношению (21) для всех натуральных $l, l<k+N=n-1$, имеет место оценка

$$
\left|\left(r_{k}(f), g_{l}\right)\right|=\left|\left(f_{N+k}, g_{l}\right)\right| \leqslant \frac{1 / 2}{(n-1)^{1 / 2}}+\frac{1 / 2}{n-1}<\left|\left(f_{n-1}, g_{n}\right)\right|=\left|\left(r_{k}(f), g_{n}\right)\right| .
$$

Следовательно,

$$
e_{k+1}(f)=g_{n},
$$


и согласно формулам $(3),(4),(15)$ и равенству $\varepsilon_{k+1}=0$

$$
\begin{aligned}
r_{k+1}(f) & =r_{k}(f)-\left(\left(r_{k}(f), e_{k+1}(f)\right)+\xi_{k+1}\right) e_{k+1}(f) \\
& =f_{n-1}-\left(\left(f_{n-1}, g_{n}\right)+\xi_{n-N}\right) g_{n}=f_{n-1}-c_{n} g_{n}=f_{n} .
\end{aligned}
$$

Используя неравенство (18), получим соотношение

$$
\left|\left(f_{n}, g_{n}\right)\right|=\left|\left(f_{n-1}-\left(\left(f_{n-1}, g_{n}\right)+\xi_{n-N}\right) g_{n}, g_{n}\right)\right|=\xi_{n-N}<\frac{1 / 2}{n^{1 / 2}}+\frac{1 / 2}{n} .
$$

Наконец, из предположения индукции следует, что для всех натуральных $l, l<n$, выполняется оценка

$$
\begin{aligned}
\left|\left(f_{n}, g_{l}\right)\right| & =\left|\left(f_{n-1}, g_{l}\right)\right| \frac{\left(f_{n}, h_{n-1}\right)}{\left(f_{n-1}, h_{n-1}\right)}=\left|\left(f_{n-1}, g_{l}\right)\right| \frac{1 / n^{1 / 2}+1 / n}{1 /(n-1)^{1 / 2}+1 /(n-1)} \\
& \leqslant\left(\frac{1 / 2}{(n-1)^{1 / 2}}+\frac{1 / 2}{n-1}\right) \frac{1 / n^{1 / 2}+1 / n}{1 /(n-1)^{1 / 2}+1 /(n-1)}=\frac{1 / 2}{n^{1 / 2}}+\frac{1 / 2}{n} .
\end{aligned}
$$

Таким образом, соотношения (21) доказаны. Для завершения доказательства теоремы 3 остается заметить, что последовательность $\left\{f_{n}\right\}_{n=N}^{\infty}$, а, значит, и последовательность остатков $\left\{r_{n}(f)\right\}_{n=0}^{\infty}$, расходится.

ДокАЗАТЕЛЬСТво тЕОремЫ 4. Положим $t_{n}=1 / \ln ^{2} n, n \in \mathbb{N}$. Теорема 1 из работы [8] утверждает, что существуют такие действительноегильбертово пространство $H$, словарь $D \subset H$ и последовательности $\left\{r_{n}\right\}_{n=0}^{\infty} \subset H,\left\{e_{n}\right\}_{n=1}^{\infty} \subset D$, что для всех целых неотрицательных $n$ справедливы соотношения

$$
\begin{gathered}
r_{n+1}=r_{n}-\left(r_{n}, e_{n+1}\right) e_{n+1}, \\
\left|\left(r_{n}, e_{n+1}\right)\right| \geqslant t_{n+1} \sup _{e \in D}\left|\left(r_{n}, e\right)\right|
\end{gathered}
$$

и, кроме того,

$$
r_{n} \nrightarrow \text { A } 0, \quad n \rightarrow \infty .
$$

Из неравенств (22) получаем, что для всех целых неотрицательных $n$ вьполняется оценка

$$
\sup _{e \in D}\left|\left(r_{n}, e\right)\right| \leqslant\left|\left(r_{n}, e_{n+1}\right)\right| \frac{1}{t_{n+1}} .
$$

Для всех $n \geqslant 0$ положим

$$
q_{n+1}=\left|\left(r_{n}, e_{n+1}\right)\right|\left(\frac{1}{t_{n+1}}-1\right)
$$

Используя оценку (24) и равенство (25), перепишем соотношение (22) следующим обра3ом:

$$
\begin{aligned}
\left|\left(r_{n}, e_{n+1}\right)\right| & \geqslant t_{n+1} \sup _{e \in D}\left|\left(r_{n}, e\right)\right|=\sup _{e \in D}\left|\left(r_{n}, e\right)\right|-\left(1-t_{n+1}\right) \sup _{e \in D}\left|\left(r_{n}, e\right)\right| \\
& \geqslant \sup _{e \in D}\left|\left(r_{n}, e\right)\right|-\left(1-t_{n+1}\right)\left|\left(r_{n}, e_{n+1}\right)\right| \frac{1}{t_{n+1}}=\sup _{e \in D}\left|\left(r_{n}, e\right)\right|-q_{n+1} .
\end{aligned}
$$


Заметим, что этот процесс может быть рассмотрен как gAWGA-разложение элемента $f:=r_{0}$ по словарю $D$ с ослабляющими последовательностями $t_{n}=1, n=1,2,3, \ldots$, $\left\{q_{n}\right\}_{n=1}^{\infty}$ и нулевьми ошибками. Согласно соотношению (23) это разложение не сходится к разлагаемому элементу. Для завершения доказательства теоремы остается оценить $q_{n}, n=1,2,3, \ldots$ Из доказательства теоремы 1 работы [8] легко может быть получено следующее утверждение: существует такое действительное число $C, C>0$, что для всех целых неотрицательных $n$ справедливо неравенство

$$
\left|\left(r_{n}, e_{n+1}\right)\right|<C \frac{t_{n+1}}{\sqrt{n+1}} .
$$

Используя равенство (25), получаем, что для всех натуральных $n$ справедлива оценка

$$
q_{n} \leqslant \frac{C}{\sqrt{n}} .
$$

3. gAWGA-разложения по ортонормированным системам. В этом пункте изучаются некоторые вопросы сходимости gAWGA-разложений с параметрами $q_{n}=0$, $t_{n}=t>0$ и $\varepsilon_{n}=0, n=1,2,3, \ldots$, по ортонормированным базисам. Оказывается, в этом случае ситуации $t=1$ и $t<1$ являются принципиально разньми. Точнее, справедливы следующие теоремы.

Теорема 5. Пусть $D$ - ортонормированный базис, $\xi_{n} \rightarrow 0$ при $n \rightarrow \infty, t_{n}=1 u$ $q_{n}=0, n=1,2,3, \ldots$. Тогда для всех әлементов $f \in H$ gAWGA-разложсение $f$ по словарю $D$ с ослабляюшими последовательностями $\left\{t_{n}\right\}_{n=1}^{\infty},\left\{q_{n}\right\}_{n=1}^{\infty}$ и оиибками $\left\{\left(0, \xi_{n}\right)\right\}_{n=1}^{\infty}$ сходится $\kappa f$.

Теорема 6. Для всех $t \in(0,1)$ существуют гильбертово пространство $H$, ортонормированный базис $D \subset H$, әлемент $f \in H$ и последовательность $\left\{\xi_{n}\right\}_{n=1}^{\infty}$ такие, что $\xi_{n} \rightarrow 0$ при $n \rightarrow \infty$, но gAWGA-разложсение $f$ по словарю $D$ с ослабляюшими последовательностями $t_{n}=t, q_{n}=0, n=1,2,3, \ldots, u$ ошибками $\left\{\left(0, \xi_{n}\right)\right\}_{n=1}^{\infty}$ расходится.

ДОКАЗАТЕЛЬСТВО ТЕОРЕМЫ 5 начнем со слеДующей леммы.

Лемма 6. Пусть $D_{1}-$ произвольный словарь, $t_{n}=1, q_{n}=0, n=1,2,3, \ldots, u$ $\left\{\xi_{n}\right\}_{n=1}^{\infty}-$ произвольная ограниченная числовая последовательность. Тогда для любого әлемента $f \in H$, любого челого неотрицательного числа $l$ и любого иелого неотрицательного числа $m$ остатки gAWGA-разложения $f$ по словарю $D_{1} c$ ослабляюшими последовательностями $\left\{t_{n}\right\}_{n=1}^{\infty},\left\{q_{n}\right\}_{n=1}^{\infty}$ и ошибками $\left\{\left(0, \xi_{n}\right)\right\}_{n=1}^{\infty}$ удовлетворяют соотношению

$$
\left\|r_{l+m}(f)\right\|^{2} \leqslant\left\|r_{l}(f)\right\|^{2}+\left|\xi_{l+m}\right|^{2}
$$

В частности, для любого натурального числа $m$ выполняется неравенство

$$
\left\|r_{m}(f)\right\|^{2} \leqslant\|f\|^{2}+\left|\xi_{m}\right|^{2} \leqslant\|f\|^{2}+\sup _{k=1,2,3, \ldots}\left|\xi_{k}\right|^{2}
$$


ДокАЗАТЕЛЬСТво. Для произвольного натурального числа $k$ из соотношений $(2)$ и $(3)$ и равенств $t_{k+1}=1, q_{k+1}=0, \varepsilon_{k}=0$ имеем

$$
\left|\left(r_{k}, e_{k+1}\right)\right|=\sup _{e \in D}\left|\left(r_{k}, e\right)\right| \geqslant\left|\left(r_{k}, e_{k}\right)\right|=\left|\xi_{k}\right| .
$$

Отсюда согласно следствию 1 из леммы 1 и равенствам $\varepsilon_{k}=0, k=l+1, l+2, \ldots, l+m$, получаем, что для всех целых неотрицательных $l$ и всех натуральных $m$ справедливо соотношение

$$
\begin{aligned}
\left\|r_{l+m}(f)\right\|^{2} & =\left\|r_{l}(f)\right\|^{2}-\sum_{k=l+1}^{l+m}\left(\left|\left(r_{k-1}, e_{k}\right)\right|^{2}-\left|\xi_{k}\right|^{2}\right) \\
& =\left\|r_{l}(f)\right\|^{2}-\left|\left(r_{l}, e_{l+1}\right)\right|^{2}+\left|\xi_{l+m}\right|^{2}-\sum_{k=l+1}^{l+m-1}\left(\left|\left(r_{k}, e_{k+1}\right)\right|^{2}-\left|\xi_{k}\right|^{2}\right) \\
& \leqslant\left\|r_{l}(f)\right\|^{2}-\left|\xi_{l+m}\right|^{2} .
\end{aligned}
$$

Лемма 6 доказана.

Перейдем непосредственно к доказательству теоремы 5 . Пусть $D=\left\{h_{n}\right\}_{n=1}^{\infty}-$ opтонормированный базис в гильбертовом пространстве $H$. Зафиксируем произвольный элемент $f \in H$ и произвольное положительное число $\varepsilon$. Без ограничения общности можно считать, что последовательность $\left\{\left|\left(f, h_{k}\right)\right|\right\}_{k=1}^{\infty}$ не возрастает.

Найдем натуральное число $K$ такое, что

$$
K\left|\left(f, h_{K}\right)\right|^{2}+\sum_{k=K+1}^{\infty}\left|\left(f, h_{k}\right)\right|^{2}<\varepsilon
$$

(отсюда, в частности, следует, что $\left.\left|\left(f, h_{K}\right)\right|^{2}<\varepsilon / K\right)$. Далее найдем натуральное число $N_{1}$ такое, что для любого $n \geqslant N_{1}$ вьполняется неравенство $\left|\xi_{n}\right|^{2}<\varepsilon / K$.

Положим $N=N_{1}+K$. Покажем, что для любого натурального $n$ такого, что $n>N$, вьполняется неравенство $\left\|r_{n}\right\|^{2}<2 \varepsilon$. Предположим сначала, что $\left\{e_{k}(f)\right\}_{k=1}^{n} \subset$ $\left\{h_{k}\right\}_{k=1}^{K}$. В этом случае при всех $k=1,2,3, \ldots, K$ будет справедлива оценка

$$
\left|\left(r_{n}, h_{k}\right)\right|<\frac{\varepsilon}{K} .
$$

Действительно, если $e_{j+1}(f)=h_{k}, 1 \leqslant k \leqslant K$, то

$$
\left|\left(r_{j+1}, h_{k}\right)\right|=\left|\xi_{j+1}\right| \text {. }
$$

Отсюда и из выбора чисел $N_{1}$ и $N$ вытекает, что, начиная с номера $j=N_{1}$, количество индексов $k$ из множества $\{1,2, \ldots, K\}$, для которых оценка $(27)$ неверна, при переходе от остатка $r_{j}$ к остатку $r_{j+1}$ будет уменьшаться на единицу, пока не достигнет нуля. Отсюда в силу соотношения (26) получаем, что

$$
\begin{aligned}
\left\|r_{n}\right\|^{2} & =\sum_{k=1}^{\infty}\left|\left(r_{n}, h_{k}\right)\right|^{2} \leqslant K \frac{\varepsilon}{K}+\sum_{k=K+1}^{\infty}\left|\left(f, h_{k}\right)\right|^{2} \\
& \leqslant K \frac{\varepsilon}{K}+K\left|\left(f, h_{K}\right)\right|^{2}+\sum_{k=K+1}^{\infty}\left|\left(f, h_{k}\right)\right|^{2}<2 \varepsilon .
\end{aligned}
$$


Рассмотрим теперь случай $\left\{e_{k}(f)\right\}_{k=1}^{n} \not \subset\left\{h_{n}\right\}_{n=1}^{K}$. Пусть $l$ - первьй из индексов, удовлетворяюший условию $e_{l+1}(f) \notin\left\{h_{n}\right\}_{n=1}^{K}$. Тогда имеет место соотношение

$$
\left(r_{l}, h_{k}\right)=\left(f, h_{k}\right), \quad k \geqslant K+1 \text {. }
$$

Из формулы (2), равенств $t_{k}=1, q_{k}=0, k=1,2,3, \ldots$, соотношения (28), а также монотонности последовательности $\left\{\left|\left(f, h_{k}\right)\right|\right\}_{k=1}^{\infty}$ получаем, что при всех $k=1,2,3, \ldots, K$ справедлива оценка

$$
\left|\left(r_{l}, h_{k}\right)\right| \leqslant\left|\left(r_{l}, e_{l+1}(f)\right)\right|=\left|\left(f, e_{l+1}(f)\right)\right| \leqslant\left|\left(f, h_{K}\right)\right| \text {. }
$$

Отсюда с учетом соотношений $(26)$ и (28) следует, что

$$
\left\|r_{l}\right\|^{2}=\sum_{k=1}^{\infty}\left|\left(r_{l}, h_{k}\right)\right|^{2} \leqslant K\left|\left(f, h_{K}\right)\right|^{2}+\sum_{k=K+1}^{\infty}\left|\left(f, h_{k}\right)\right|^{2}<\varepsilon .
$$

Из последнего неравенства, выбора числа $N_{1}$ и леммы 6 получаем, что

$$
\left\|r_{n}\right\|^{2} \leqslant\left\|r_{l}\right\|^{2}+\left|\xi_{n}\right|^{2}<\varepsilon+\frac{\varepsilon}{K}<2 \varepsilon .
$$

Таким образом, для любого $\varepsilon>0$ существует такое натуральное $N$, что для всех натуральных $n, n>N$, справедливо неравенство $\left\|r_{n}\right\|^{2}<2 \varepsilon$. Следовательно, $r_{n} \rightarrow 0$ при $n \rightarrow \infty$, откуда сразу вытекает утверждение теоремы.

ДОКАЗАТЕЛЬСТВО ТЕОРЕмЫ 6. Пусть $H$ - произвольное сепарабельное бесконечномерное гильбертово пространство, $D=\left\{h_{n}\right\}_{n=1}^{\infty}-$ произвольньй ортонормированный базис в $H$. Рассмотрим элемент $f \in H$, имеющий следуюшие координаты относительно базиса $D$ :

$$
f=\left(\frac{1}{2}, \frac{1}{2}, \frac{1}{4}, \frac{1}{4}, \frac{1}{4}, \frac{1}{4}, \frac{1}{8}, \frac{1}{8}, \frac{1}{8}, \frac{1}{8}, \frac{1}{8}, \frac{1}{8}, \frac{1}{8}, \frac{1}{8}, \ldots\right) .
$$

Будем строить последовательность абсолютных ошибок $\left\{\xi_{n}\right\}_{n=1}^{\infty}$ и одновременно смотреть на остатки gAWGA-разложений элемента $f$ по словарю $D$.

Выберем в качестве $e_{1}(f)$ элемент $h_{1}$ и положим $\xi_{1}=-1 /(2 t)$. В таком случае в качестве $e_{2}(f)$ можно выбрать элемент $h_{2}$. Положим $\xi_{2}=-1 /(2 t)$, тогда

$$
r_{2}(f)=\left(\frac{1}{2 t}, \frac{1}{2 t}, \frac{1}{4}, \frac{1}{4}, \frac{1}{4}, \frac{1}{4}, \frac{1}{8}, \frac{1}{8}, \frac{1}{8}, \frac{1}{8}, \frac{1}{8}, \frac{1}{8}, \frac{1}{8}, \frac{1}{8}, \ldots\right) .
$$

Далее возьмем $e_{3}(f)=h_{1}, \xi_{3}=-1 /\left(2 t^{2}\right), e_{4}(f)=h_{2}, \xi_{4}=-1 /\left(2 t^{2}\right)$. Остаток $r_{4}(f)$ будет иметь координаты

$$
r_{4}(f)=\left(\frac{1}{2 t^{2}}, \frac{1}{2 t^{2}}, \frac{1}{4}, \frac{1}{4}, \frac{1}{4}, \frac{1}{4}, \frac{1}{8}, \frac{1}{8}, \frac{1}{8}, \frac{1}{8}, \frac{1}{8}, \frac{1}{8}, \frac{1}{8}, \frac{1}{8}, \ldots\right) .
$$

Продолжим аналогичньм образом изменять первые две координаты остатков до тех пор, пока эти координаты не станут больше единицы. Пусть $n_{1}$ - это то самое число, для которого

$$
r_{n_{1}}=\left(a, a, \frac{1}{4}, \frac{1}{4}, \frac{1}{4}, \frac{1}{4}, \frac{1}{8}, \frac{1}{8}, \frac{1}{8}, \frac{1}{8}, \frac{1}{8}, \frac{1}{8}, \frac{1}{8}, \frac{1}{8}, \ldots\right),
$$


$a>1$, но $a t \leqslant 1$. Далее, возьмем в качестве $e_{n_{1}+1}$ элемент $h_{1}$, положим $\xi_{n_{1}+1}=0$, возьмем в качестве $e_{n_{1}+2}$ элемент $h_{2}$ и положим $\xi_{n_{1}+2}=0$. Имеем

$$
r_{n_{1}+2}=\left(0,0, \frac{1}{4}, \frac{1}{4}, \frac{1}{4}, \frac{1}{4}, \frac{1}{8}, \frac{1}{8}, \frac{1}{8}, \frac{1}{8}, \frac{1}{8}, \frac{1}{8}, \frac{1}{8}, \frac{1}{8}, \ldots\right) .
$$

Далее будем изменять третью, четвертую, пятую и шестую координаты остатков. Возьмем в качестве $e_{n_{1}+3}$ элемент $h_{3}$ и положим $\xi_{n_{1}+3}=-1 /(4 t)$, аналогично положим $e_{n_{1}+4}=h_{4}, \xi_{n_{1}+4}=-1 /(4 t), e_{n_{1}+5}=h_{5}, \xi_{n_{1}+5}=-1 /(4 t), e_{n_{1}+6}=h_{3}, \xi_{n_{1}+6}=$ $-1 /(4 t)$. Остаток $r_{n_{1}+6}(f)$ имеет координаты

$$
r_{n_{1}+6}(f)=\left(0,0, \frac{1}{4 t}, \frac{1}{4 t}, \frac{1}{4 t}, \frac{1}{4 t}, \frac{1}{8}, \frac{1}{8}, \frac{1}{8}, \frac{1}{8}, \frac{1}{8}, \frac{1}{8}, \frac{1}{8}, \frac{1}{8}, \ldots\right) .
$$

Следуюшие четыре шага сделаем следуюшим образом: $e_{n_{1}+7}=h_{3}, \xi_{n_{1}+7}=-1 /\left(4 t^{2}\right)$, $e_{n_{1}+8}=h_{4}, \xi_{n_{1}+8}=-1 /\left(4 t^{2}\right), e_{n_{1}+9}=h_{5}, \xi_{n_{1}+9}=-1 /\left(4 t^{2}\right), e_{n_{1}+10}=h_{3}, \xi_{n_{1}+10}=$ $-1 /\left(4 t^{2}\right)$. После этих шагов остаток примет вид

$$
r_{n_{1}+10}(f)=\left(0,0, \frac{1}{4 t^{2}}, \frac{1}{4 t^{2}}, \frac{1}{4 t^{2}}, \frac{1}{4 t^{2}}, \frac{1}{8}, \frac{1}{8}, \frac{1}{8}, \frac{1}{8}, \frac{1}{8}, \frac{1}{8}, \frac{1}{8}, \frac{1}{8}, \ldots\right) .
$$

Продолжим аналогичньм образом изменять координаты остатков с третьей по шестую до тех пор, пока эти координаты не станут больше $1 / 2$. Пусть $n_{2}$ - то самое число, для которого

$$
r_{n_{1}}=\left(0,0, b, b, b, b, \frac{1}{8}, \frac{1}{8}, \frac{1}{8}, \frac{1}{8}, \frac{1}{8}, \frac{1}{8}, \frac{1}{8}, \frac{1}{8}, \ldots\right),
$$

$b>1 / 2$, но $b t \leqslant 1 / 2$. Далее положим $e_{n_{2}+1}=h_{3}, \xi_{n_{2}+1}=0, e_{n_{2}+2}=h_{4}, \xi_{n_{2}+2}=0$, $e_{n_{2}+3}=h_{5}, \xi_{n_{2}+3}=0, e_{n_{2}+4}=h_{6}, \xi_{n_{2}+4}=0$ и перейдем к следующему блоку. Используя ту же технику, будем изменять следующие восемь координат остатков (с седьмой по четырнадцатую) до тех пор, пока эти координаты не станут больше $1 / 3$. Обозначим номер последнего сделанного шага через $n_{3}$. Далее сделаем восемь шагов с нулевыми ошибками и перейдем к четвертому блоку координат (координаты с пятнадцатой по тридцатую), затем к пятому (координаты с номерами 31-62) и так далее (будем увеличивать координаты $m$-го блока до тех пор, пока эти координаты не станут больше $1 / m$, а затем обнулим координаты этого блока). Пусть $n_{m}-$ номер последнего шага при увеличении абсолютных величин координат $m$-го блока.

Построение закончено. Остается показать, что $\xi_{n} \rightarrow 0, n \rightarrow \infty$, и что последовательность остатков $\left\{r_{n}(f)\right\}_{n=1}^{\infty}$ расходится. Первое утверждение следует из того, что наибольшая ошибка при изменении $m$-го координатного блока не превосходит $1 /(m t)$, второе утверждение следует из неравенства

$$
\left\|r_{n_{m}}(f)\right\|^{2}>\frac{2^{m}}{m^{2}}, \quad m=1,2,3, \ldots
$$

ЗАмЕчАниЕ 1. Следующее утверждение дополняет теорему 6. 
В условиях теоремы 6 существует такая строго возрастающая последовательность натуральных чисел $\left\{n_{k}\right\}_{k=1}^{\infty}$, что $r_{n_{k}}(f) \rightarrow 0, k \rightarrow \infty$, или, әквивалентно,

$$
\sum_{j=1}^{n_{k}} \widehat{f}_{j} e_{j} \rightarrow f, \quad k \rightarrow \infty .
$$

Доказательство этого утверждения аналогично доказательству теоремы 5.

ЗАмЕчАниЕ 2. В теоремах 2 и 5 предполагается, что рассматриваемые gAWGAразложения осуществимы.

\section{СПИСОК ЦИТИРОВАННОЙ ЛИТЕРАТУРЫ}

[1] Gribonval R., Nielsen M. Approximate weak greedy algorithms // Adv. Comput. Math. 2001. V. 14. № 4. P. 361-378.

[2] Temlyakov V. N. Weak greedy algorithms // Adv. Comput. Math. 2000. V. 12. № 2-3. P. 213-227.

[3] Friedman J.H., Stueuzle W. Projection pursuit regression // J. Amer. Statist. Assoc. 1981. V. 76. P. 817-823.

[4] Mallat S., Zhang Z. Matching pursuit with time-frequency dictionaries // IEEE Trans. Signal Process. 1993. V. 41. №12. P. 3397-3415.

[5] Jones L.K. On a conjecture of Huber concerning the convergence of PP-regression // Ann. Statist. 1987. V. 15. P. 880-882.

[6] Jones L. A simple lemma on greedy approximation in Hilbert space and convergence rates for projection pursuit regression and network training // Ann. Statist. 1992. V. 20. P. 608-613.

[7] Rejtő L., Walter G. G. Remarks on ptojection pursuit regression and density estimation // Stochastic Anal. Appl. 1992. V. 10. P. 213-222.

[8] Лившиц Е. Д., Темляков В.Н. О сходимости слабого гриди-алгоритма // Тр. МИАН. 2001. T. 232. C. 236-247.

[9] Temlyakov V.N. A criterion for convergence of weak greedy algorithms // Preprint at http://www.math.sc.edu/ ${ }^{\sim}$ imip/00.html.

[10] Galatenko V. V., Livshitz E. D. On the convergence of approximate weak greedy algorithms // East J. Approx. 2003. V. 9. №1. P. 43-49. 